\title{
15 Jahre «helvetisches Schleudertrauma»
}

Gerhard Jenzer

Korrespondenz:

Dr. med. Gerhard Jenzer Spezialarzt für Neurologie FMH Försterstrasse 8

CH-4900 Langenthal

Tel. 0629227969

Fax 0629237487

gejenzer@bluewin.ch
Die Malediktion «Schleudertrauma» führt im spezifischen soziokulturellen Umfeld Schweiz zu gravierenden Auswirkungen für die Betroffenen und die gesamte Gesellschaft. Die dieser Unfallart folgende Phänomenologie bedarf neben einer eindeutigen faktischen Klärung anhand der biomechanischen und medizinischen Gegebenheiten einer Interpretation im biopsychosozialen Kontext.

\section{Richterlicher Sachentscheid}

Im Aufsatz vom 26. Juni 1991 [1] wird im Zusammenhang mit dem Salanitri-Urteil des EVG [2] erstmals darauf hingewiesen, dass die Schweiz im Umgang mit Folgen von Auffahrkollisionen und distorsioneller Belastung der Nacken-KopfRegion (ohne nachweisbare Organschädigung) einen eigenen Weg geht. Nicht mehr anhand der in diesem Zusammenhang unergiebigen medizinisch objektivierbaren Sachverhalte soll künftig über die zu bejahende kausale Zuordnung solcher Unfallfolgen entschieden werden, sondern nach höchstrichterlicher Wertung eines subjektiven, «typischen» Beschwerdebildes.

Dass dabei seinerzeit - am falschen Exempel statuiert - die faktischen Voraussetzungen nicht einmal demjenigen des so überaus häufigen «Whiplash»-Mechanismus entsprochen hatten, sondern dem elementar unterschiedlichen Unfall eines gegen eine Böschung gestürzten Motorradfahrers, mit Kopfanprall und «mild traumatic brain injury», ist heute nur noch von anekdotischem oder allenfalls epistemologischem Interesse.

\section{Standardfall}

«Whiplash» oder «Schleudertrauma» ist bekanntlich das überraschende meist von hinten Angestossenwerden mit Akzeleration im Bereich der dadurch potentiell einer Distorsionswirkung unterworfenen Nackenorgane, ohne Kontakt des Kopfes ausser an der (im Gegensatz zur Originalbeschreibung nun vorhandenen) Kopfstütze. Als Verletzungsfolge resultieren möglicherweise eine Gefügestörung der Halswirbelsäule und eine Zerrung der umgebenden Weichteile. Inzwischen ist durch zahlreiche erstklassige Untersuchungen zumindest für die zahlenmässig weit überwiegende Kategorie der im «low impact»Bereich Betroffenen (s.u.) belegt, dass keinerlei Organschäden zurückbleiben, weder an den
Strukturen des Nacken-Kopf-Bereichs noch insbesondere am Gehirn. Entweder treten überhaupt keine Beschwerden auf, oder der normale Heilungsverlauf der distorsionellen Folgen (Stauchung, Zerrung, hypothetische «Mikroläsionen» im Bereich der Halswirbelsäule) spielt sich meistens über lokale Schmerzzustände während Tagen bis wenigen Wochen ab. Diese Tatsachen halten einer ernsthaften Prüfung stand. Zerreden mit Einzelbeispielen, wo sich gegenteilige Befunde oder Verläufe einstellten, führen zur Verunklarung, da vollständige individuelle Daten aller jeweiligen Umstände vorweg fehlen. Der Diskurs über das Gros der «Normalfälle» im klinischen Alltag ist notwendig und darf beanspruchen, nicht vereitelt zu werden.

Wenn dem Kollisionsunfall nicht die gewöhnlich zu erwartende Heilung folgt, verbleibt eine sich oft weiter ausbreitende Schmerzsymptomatik an Nacken und/oder Kopf im häufigen, jedoch fakultativen Verbund zum «typischen» Beschwerdebild.

Charakter und Entstehungsbedingungen dieser Erscheinungen sollen hier betrachtet werden.

\section{Nacken-Kopf-Schmerz}

In der IHS-Klassifikation («Headache attributed to whiplash injury», 5.3/5.4) [3] wird die Schmerzsymptomatik nach «Whiplash» neuerdings wahrgenommen und zum allgemein anerkannten Sachverhalt erhoben. Gegen die Katalogisierung ist allerdings sofort einwendbar, dass sie einer individuellen klinischen Wirklichkeit widerspricht; dafür spricht jedoch, dass jede weitere Diskussion auf wissenschaftlicher Grundlage obligat einer begrifflichen Einigung über die in den zitierten Studien verwendeten Diagnosen bedarf. Sowohl dem akuten wie dem chronischen Schmerz, der dem Folgezustand zugeordnet wird, sind gemäss IHS keinerlei «typischen Charakteristika» eigen. Aus der Art dieser Schmerzsymptomatik können unter dieser Betrachtungsweise also keine verlässlichen Schlüsse auf eigentliche Ursachen gezogen werden. Die Pathogenese bleibt demzufolge ebenfalls ungeklärt und enthebt im gleichen Zusammenhang folgerichtig bis auf weiteres von der Argumentation über ein relevantes «organisches» Substrat nach «Whiplash Injury». Unterscheidbar gemäss IHS ist jedoch ein «zervikogener Kopfschmerz» (11.2.1), der auf eine distinkte Quelle im Nacken- 
bereich bzw. auf eine identifizierte Ursache bezogen werden kann und dessen Verschwinden nach lokaler Therapie als retrospektiv diagnostisch gilt. Dieses Konzept steht im Einklang mit der definitorischen Annahme, dass bei Verletzungsgraden nach QTF (Quebec Task Force) von 0 bis 2 [4] keine bleibende, nachweisliche strukturelle Schädigung der Halswirbelsäule vorangegangen ist.

\section{«Typisches» Beschwerdebild}

Typisch heisst bei weitem nicht exklusiv kennzeichnend oder pathognomonisch.

Im Bericht der Schweizerischen Neurologischen Gesellschaft [5] wird festgehalten, dass das Beschwerdebild ohne initial festgestellte Bewusstseinsstörung (gleichzeitig eingetretenes «Mild traumatic brain injury») nicht als Folge einer Hirnschädigung nachgewiesen ist. Dahin zielende Untersuchungen wie MRI des Schädels, PET und SPECT sowie Neuropsychologie haben diesen Nachweis auch seither nicht erbracht. Hier drängt sich der Hinweis auf, dass sich eine Theorie nicht durch ihre Unwiderlegbarkeit, sondern durch den Beweis bewahrheitet.

Der Inhalt des «typischen» Beschwerdebildes weitet sich ungünstigenfalls im Verlauf von Monaten bis Jahren aus. Im Wissen um die unter regelhaften Umständen durch nichts aufzuhaltende Regeneration und Anpassung zur physiologischen Normalität ist eine solche Fehlentwicklung auf «organischer» Grundlage unerklärbar. Vorauszusetzen ist allerdings die Abwesenheit eines neu und gleichzeitig in Gang kommenden prozesshaften Geschehens (als Komorbidität). Aus dieser Disposition folgt die pragmatische Annahme, dass weitere Einflüsse im Spiele sind. Das dichotome Soma-Psyche-Modell vermag diese Komplexität allerdings nicht zu fassen und gehört, zumindest für die Medizin, in die Asservatenkammer.

\section{Psychophysische Syndrome}

Mit dem Aufkommen von Eisenbahnen und Reiseversicherungen im viktorianischen England nahm die sogenannte "Railway spine» eine epidemische Ausbreitung. Zugspassagiere klagten nach Unfällen über multiple Symptome, die als körperlich bedingte «nervöse Irritation» verstanden wurden. Unter der Anfang des letzten Jahrhunderts folgenden, offenbar überzeugenderen Erklärung dieser Beschwerden mit dem Neurosebegriff, ursprünglich ebenfalls als «organisch» aufgefasst, verschwand das Phänomen.

Ein weiterer Blick zurück ist hilfreich, indem die «typische» Vielfalt der noch als «organisch» angesehenen Symptome Mitte des 19. Jahrhunderts in Frankreich mit «Neurasthenie» einen Namen fand. Die Bezeichnung erfuhr in kurzer Zeit eine enorme Resonanz, um dann etwa 1930 zu verblassen. Abgesehen von Psychosen, und neben «Hysterie», betraf vordem Neurasthenie die häufigste psychiatrische Diagnose und sie gab auch oft Anlass zu Hospitalisation im somatischen Bereich. S. Freud deutete sie mit eigenwilliger kausaler Erklärung als «Aktualneurose».

In den letzten Jahrzehnten wurde Neurasthenie im Grenzbereich zu neuen Diagnosen, wie Chronic-Fatigue-Syndrom, Fibromyalgie, atypische Depression und somatoforme Dysfunktion bzw. Schmerzkrankheit, debattiert.

Bemerkenswert enthielt schon die erste eingehende Schweizer Studie zu «Whiplash Injury» [6] den Hinweis auf Neurasthenie, als Bezeichnung für das die Schmerzsymptomatik umgebende komplexe Beschwerdebild. Die Symptomkonstellation Kopfschmerz, Müdigkeit, Schlafstörung, Konzentrationsstörung, Vergesslichkeit und Reizbarkeit nach «Whiplash Injury» erwies sich im Vergleich mit der Befindlichkeit bei «multiple chemical sensitivities», «chronic fatigue syndrome» und Fibromyalgie als frappant ähnlich [7]. In diesem Licht betrachtet ist der Beizug des von Neurasthenie nicht unterscheidbaren «typischen» Beschwerdebildes als Beweis für eine durch den «Whiplash»-Mechanismus verursachte «organische» Verletzung unwissenschaftlich, willkürlich.

\section{Vulnerabilitätsgrenzen}

Ohne die Annahme von Vulnerabilitätsgrenzen ist die Evolution nicht vorstellbar. Bestimmte Grössen von Belastung müssen aushaltbar sein, ohne dass es zu Verletzungen kommt, was ohne weiteres einleuchtet. Zur Erfassung verletzungsrelevanter Wirkungen bei Kollisionsunfällen entspricht die von den ursprünglichen Fahrgeschwindigkeiten oft stark abweichende Geschwindigkeitsänderung Delta-v dem aktuell am besten geeigneten Parameter. Die nachträgliche technische Bestimmung insbesondere dieser Grösse und deren biomechanische Interpretation bezüglich Verletzungspotential gewinnen zunehmend an Bedeutung. Bei einer Heckkollision mit einem Delta-v unterhalb von $10 \mathrm{~km} / \mathrm{h}$ als untere Toleranzgrenze («low impact») spricht mehr gegen als für (überwiegende Wahrscheinlichkeit) das Eingetretensein einer HWS-distorsionellen Schädigung. Bis zu einer oberen Toleranzgrenze von Delta-v $15 \mathrm{~km} / \mathrm{h}$ kann, muss aber nicht, eine solche eingetreten sein. Selbst höhere Werte von Delta-v bedingen keineswegs zwangs- 
läufig Verletzungen [8]. Diese Annahmen werden denn auch in der deutschen Rechtsprechung umgesetzt.

Spätestens in Kenntnis von simulierten Crashversuchen (Delta- $v=0$ ) [9] mit freiwilligen Versuchspersonen, die unter den gleichen Symptomen litten, wie wenn ein Zusammenstoss tatsächlich stattgefunden hätte, sind Überlegungen am Platze, die neben der «organischen» Sichtweise andere Erklärungsmodelle nicht nur zulassen, sondern erfordern.

\section{Soziokulturelles Phänomen}

Wir haben mittlerweile von einer nicht wegzuredenden länderspezifischen Diskrepanz bezüglich Schadenhäufigkeit und Fallkosten auszugehen [10]. Wenn in Frankreich 3\% sämtlicher Personenschäden auf das Konto von HWS-Verletzungen gehen, sind es in der Schweiz 33\%. Der Schadenaufwand pro HWS-Verletzung zeigt das Verhältnis von $€ 2625$ zu $€ 35$ 000, entsprechend einem Betrag, mit welchem die Schweiz gegenüber der zweitteuersten Nation (Niederlande) bei einer Differenz von $€ 18500$ noch deutlich Abstand nimmt. Nur mit gemischter Freude ist ausserdem zu beobachten, dass die welsche Schweiz, offenbar in einer gewissen Annäherung an die französische Mentalität, in Sachen «Whiplash» wesentlich geringere Kosten generiert als die Deutschschweiz. Die Verletzungsart fand sich besonders häufig in den Städten Zürich, Basel und Luzern [11]. Peinlich, wenn wir annehmen müssten, dass regionalspezifische Erzeuger des grösseren Aufwandes zugleich fachlich besser befähigt seien, mit dem Szenario der kostspieligen Langzeitfolgen umzugehen.

Die inkonsistente Beziehung zwischen «Whiplash»-Symptomatik und eigentlicher Verletzung, bei weltweit höchst unterschiedlicher Prävalenz nachfolgender Beschwerden, unterstreicht den Stellenwert der sozialen und kulturellen Einflüsse [12]. Um zunächst Europa nochmals zu betrachten, kennen Griechenland oder Litauen [13] noch so gut wie keine Folgen von «Whiplash». Länger bekannt ist das hohe Vorkommen von solchen in Australien, im Gegensatz zur sehr niedrigen Prävalenz in Singapur [14], wobei ähnlich wie zwischen Frankreich und der Schweiz wohl eher auf kulturelle als auf logistisch-technische Unterschiede abzustellen ist.

Insgesamt erscheint nicht plausibel, dass bei globalem Vorkommen von Automobilverkehr und nach gleichartigen Unfallmechanismen (Newton fährt überall mit), biophysikalisch erklärbar, wesentlich abweichende Organschäden eintreten sollten, oder dass die ärztliche Diagnostik heute noch dermassen ins Gewicht fallende
Qualitätsunterschiede aufweist. Vielmehr erscheint als geradezu zwingend, dass soziokulturelle Hintergründe das Ergebnis prägen.

\section{Salutogenes vs. pathogenes Management} An Selbstkritik ist nicht vorbeizukommen. Wem die Kassandrarufe nicht gelten, wird es leicht merken. Kann den Betroffenen im Anschluss an eine sachgerechte Untersuchung eröffnet werden, dass ein Zustand nach Whiplash im QTFGrad 0 bis 2 (keine neurologischen Ausfälle, keine HWS-Frakturen) vorliegt, was in den allermeisten Fällen zutrifft, so kommt es zur wahrscheinlich schon entscheidenden Weichenstellung. Neben den üblichen Massnahmen im geringsten erforderlichen Ausmass zählt weit mehr die enorme Macht der Persuasion, sei es im guten oder im schlechten Sinne. Im Wissen um die Harmlosigkeit, nicht etwa der Geringheit der Erstsymptome wird die wahrhaft vermittelte Aussicht auf baldige Besserung helfen. Das Beibehalten der positiven Grundhaltung auch bei längeren Heilungsverläufen ist nicht nur sachgerecht, sondern eigentliche Pflicht und Schuldigkeit. Verunfallte in ihrer biopsychosozialen Einzigartigkeit wahrzunehmen und zugleich sachgerecht medizinisch zu unterstützen, verspricht Erfolg.

Hingegen wird vorauseilendes Ankünden von Schmerzen und «Hirnschaden» Resignation und Nachvollzug des Vorausgesagten begünstigen. Ungerichtete, vielfältige diagnostische Suche nach einer Erklärung von «Schmerz», mit Anhäufung von Untersuchungsresultaten und Meinungen, kann bei Betroffenen kaum zu etwas anderem als zur vertieften Einsicht und entsprechenden Empfindung des Geschädigtseins führen. Das Gleiche ist von endlosen Behandlungen ohne realistischen Horizont zu erwarten. Geradezu vernichtend ist ein den tatsächlichen Verhältnissen widersprechendes Verdikt «Hirnschaden», mit einer über Monate oder gar Jahre vorgeplanten Behandlungsdauer und Erwerbseinschränkung. Dieses kommt tatsächlich und immer öfter auf dem mit undurchsichtigen Interesselagen übersäten, reichlich genährten Boden eines vertrackten Zusammenspiels von medizinischen, psychologischen, anwaltlichen, laienorganisatorischen und richterlichen Instanzen sowie schliesslich Versicherungen unter unser aller Augen vor. Wie man leider annehmen muss, werden so konzertierte Verfahren ohne dezidiertes Gegensteuern zu einem sich festigenden Bestandteil unserer ( «Schleudertrauma»-)Kultur - und damit für «Whiplash»Beschwerden, planetarisch weit entfernt vom biomechanischen Auftakt, selbst ursächlich. 


\section{Literatur}

1 Jenzer G. Das Sagen in der Konfusion um das Schleudertrauma. Schweiz Ärztezeitung 1991; 72:1103-4.

2 Eidgenössisches Versicherungsgericht. Urteil vom 4. Februar 1991.

3 Headache Classification Subcommittee of the International Headache Society. The International Headache Classification of Headache Disorders. $2^{\text {nd }}$ edition. Cephalalgia 2004;24(Suppl 1):1-160.

4 Spitzer WO, Skovron ML, Salmi LR, Cassidy JD, Duranceau J, Suissa S, et al. Scientific Monograph of the Quebec Task Force on Whiplash-Associated Disorders: Redefining «Whiplash» and its Management. Cassidy JD, editor. 1S-73S. Quebec: Quebec Task Force on Whiplash-Associated Disorders; 1995.

5 Schnider A, Annoni JM, Dvorak J, Ettlin T, Gütling E, Jenzer $\mathrm{G}$, et al. Beschwerdebild nach kraniozervikalem Beschleunigungstrauma («whiplash-associated disorder»). Bericht der Kommission «Whiplashassociated disorder» der Schweizerischen Neurologischen Gesellschaft. Schweiz Ärztezeitung 2000; 81(39):2218-20.

6 Wiesner H, Mumenthaler M. [Whiplash injuries of the cervical spine. A catamnestic study] Arch Orthop Unfallchir 1975;81:13-36.

7 Jenzer G. Klinische Aspekte und neurologische Begutachtung beim Zustand nach Beschleunigungsmechanismus an der Halswirbelsäule. Nervenarzt 1995;66:730-5.
8 Eisenmenger W. Die Distorsion der Halswirbelsäule - Anmerkungen zur Rechtsprechung aus biomechanischer und rechtsmedizinischer Sicht. Festschrift anlässlich des 65. Geburtstages von Generalbundesanwalt Kay Nehm, 2. Februar 2006.

9 Castro WH, Meyer SJ, Becke ME, Nentwig CG, Hein MF, Ercan BI, et al. No stress - no whiplash? Prevalence of «whiplash» symptoms following exposure to a placebo rear-end collision. 9. Int J Legal Med 2001;14:316-22.

10 Schadenhäufigkeit und Schadenaufwand bei leichten Verletzungen der Halswirbelsäule Vergleichende Studie (2004). Bruxelles: Comité Européen des Assurances; 2006.

11 Zaugg A. Traumatisme de la colonne vertébrale. Schweizer Versicherung 2001. S. 38-41.

12 Solomon S. Chronic post-traumatic neck and head pain. Headache 2005;45:53-67.

13 Schrader H, Obelieniene D, Bovim G, Surkiene D, Mickeviciene D, Miseviciene I. Natural evolution of late whiplash syndrome outside the medicolegal context. Lancet 1996;347:1207-11.

14 Balla JI. The late whiplash syndrome: a study of an illness in Australia and Singapore. Culture Med Psychiatry 1982;6:191-210. 